\title{
Drying of a liquid droplet suspended in its own vapour
}

\author{
$\begin{array}{ll}\text { G. J. Oberman* } & \text { T. W. Farrell }\end{array} \quad$ E. Sizgek ${ }^{\ddagger}$
}

(Received 2 December 2004, revised 18 October 2005)

\begin{abstract}
We consider the spray drying of colloidal solutions or sols, a process that leads to the production of nanoporous powders, which are of importance in numerous manufacturing applications. An initial model of this process is formulated by considering the evaporation of a liquid droplet suspended in its own vapour. Mass, momentum, and energy balances are given for the liquid and vapour phases of the problem. Perturbation analysis shows that the system is effectively isobaric, and it is shown that surface tension may be neglected. The subsequent moving boundary problem is solved numerically and the results of this process are presented.
\end{abstract}

*School of Mathematical Sciences, QUT, Brisbane, Australia. mailto:g.oberman@student.qut.edu.au

$\dagger$ School of Mathematical Sciences, QUT, Brisbane, Australia.

${ }^{\ddagger}$ Materials Division, ANSTO, Lucas Heights, Australia.

See http://anziamj.austms.org.au/V46/CTAC2004/Ober for this article, (c) Austral. Mathematical Soc. 2005. Published November 5, 2005. ISSN 1446-8735 


\section{Contents}

1 Introduction

C1156

2 Model Development

C1157

2.1 Model Equations . . . . . . . . . . . . . . . . . . . C1158

2.2 Perturbation Analysis . . . . . . . . . . . . . . C1161

3 Numerical Techniques

C1164

4 Results and Conclusions

C1165

References

C1167

\section{Introduction}

The spray drying of colloidal solutions, or sols, is of importance in numerous manufacturing applications [6]. One such application is the production of nanoporous ceramic powders. Our overall aim is to develop models of this process in order to determine how changes in the chemistry within individual sol droplets, and in the drying conditions, alter the morphology and characteristics of the resulting powders. In this paper we present an initial model for the spray drying process in which we consider a liquid droplet evaporating within an environment of its own vapour.

A number of simplified models of spray drying have been considered. Sirignano [12] discussed the processes of droplet evaporation in the context of fuel combustion. Whilst he formulated quite complex models of the process, he made little mention of such evaporation concepts as vapor pressure or surface tension. Van der Lijn [13] modelled the spray drying of liquid foods. However, he made numerous oversimplifications, including neglecting the thermal distributions in the droplet. Sano and Keey [10] considered 
the formation of hollow sphere morphologies during the spray drying of sol droplets. However, they assumed a priori that a bubble will form inside the droplet without proof that this is the cause of the hollow sphere phenomenon.

The spray drying of sols involves four distinct phases. First, the atomised colloidal solution is released into the drying chamber. Second, once in the chamber, the droplets evaporate until colloid at the surface solidifies to form a crust. Third, liquid is evaporated through the crust and this causes the crust to thicken. Finally, when most of the liquid has been evaporated, the microsphere is heated until it exits the drying chamber. This process leads to a variety of morphologies, the most desirable being a solid sphere. Less desirable morphologies include tori and hollow spheres.

As noted earlier, we simplify the above drying process to one in which a single droplet, containing liquid, but no colloid, is being dried in a quiescent atmosphere that contains only the liquid in vapour form. Thus we only consider the first two drying phases mentioned above, with the second phase continuing until the droplet is fully evaporated. There are two reasons for modelling this simplified process. First, it provides confirmation of the equations that will be used in the more complex models that follow from this work, and, second, it allows contemplation of the method by which the moving boundary can be managed numerically.

We now consider the derivation of the model equations describing this process.

\section{Model Development}

Model Assumptions: In order to facilitate the development of a mathematical model that describes the physical system introduced above, the following simplifying assumptions are made: 
1. The liquid phase is incompressible.

2. The gas phase is ideal, inviscid, and infinite.

3. Both the liquid and gas phases are homogeneous/pure.

4. There are no outside forces acting upon the system.

5. The entire system is spherically symmetric.

6. The gas phase has significantly lower density than the liquid phase.

7. Each phase is initially in equilibrium internally.

Many, if not all, of these assumptions are standard and have been adopted, often implicitly, in many previous models of liquid drying, both in homogeneous [5, e.g.] and heterogeneous [11, e.g.] atmospheres. In particular, Assumptions 1, 3, 4 and 7 are due to the physical setup of the model; Assumptions 2 and 5 simplify the model further without oversimplifying the physics of the process being modelled; and Assumption 6 is often left unstated, yet is essential for proper thermodynamic behaviour.

As a result of the above assumptions, the following equations are formulated.

\subsection{Model Equations}

Inside the droplet: Given Assumption 1, the density of the liquid phase is constant, and the liquid phase velocity is zero. Therefore, the heat balance equation within the droplet is [1]

$$
\rho_{l} \hat{C}_{l} \frac{\partial T_{l}}{\partial t}=\frac{1}{r^{2}} \frac{\partial}{\partial r}\left(r^{2} \lambda_{l} \frac{\partial T_{l}}{\partial r}\right),
$$


where $\rho$ is the density, $\hat{C}$ is the constant volume specific heat, $T$ is the temperature, $\lambda$ is the thermal conductivity, $r$ is the radial coordinate, and $t$ is the temporal coordinate. The subscript $l$ indicates that the variable is in the liquid phase.

Initially, by Assumption 7, the temperature within the droplet is uniformly at $T_{0}$. Furthermore, at the centre of the droplet, symmetry requires

$$
\frac{\partial T_{l}}{\partial r}=0 .
$$

Outside the droplet: Noting Assumption 2, the ideal gas law may be applied, namely [9]

$$
p_{v}=C_{v} \bar{R} T_{v},
$$

where $p$ is the pressure, $C$ is the concentration, and $\bar{R}$ is the universal gas constant. The subscript $v$ indicates that the variable is in the vapour phase.

Noting Assumption 3, a material balance outside the droplet yields [1]

$$
\frac{D C_{v}}{D t}=-\frac{C_{v}}{r^{2}} \frac{\partial}{\partial r}\left(r^{2} u_{v}\right)
$$

where $u$ is the radial velocity, and $D / D t$ represents the material derivative [9].

Conservation of momentum in the gas phase [1] is simplified using the continuity equation, Assumption 2 and Assumption 4 to

$$
C_{v} \frac{D u_{v}}{D t}=-\frac{1}{m_{m}} \frac{\partial p_{v}}{\partial r}
$$

where $m_{m}$ is the molar mass of the substance.

The spherically symmetric energy balance equation is [1]

$$
m_{m} C_{v} \hat{C}_{v} \frac{D T_{v}}{D t}=\frac{1}{r^{2}} \frac{\partial}{\partial r}\left(r^{2} \lambda_{v} \frac{\partial T_{v}}{\partial r}\right)-\frac{p_{v}}{r^{2}} \frac{\partial}{\partial r}\left(r^{2} u_{v}\right)
$$


Initially, the concentration, the temperature, and the velocity of the gas are assumed to be uniformly $C_{\infty}, T_{\infty}$ and 0 , respectively. Furthermore, at a sufficiently large distance from the droplet, the temperature and concentration are assumed to be $T_{\infty}$ and $C_{\infty}$, respectively, for all times.

At the surface of the droplet: The liquid and gas phases are in thermal contact at the surface, and thus continuity of temperature applies [5]:

$$
T_{v}=T_{l}
$$

at the surface of the droplet.

All thermal energy conducted into the droplet from the gas is used for either sensible heating of the droplet, or the supply of the latent heat energy to evaporating material. As such, the heat flux surface condition is formulated as [2]

$$
\lambda \frac{\partial T_{v}}{\partial r}=\lambda_{l} \frac{\partial T_{l}}{\partial r}-L \rho_{l} \dot{R}
$$

where $L$ is the latent heat of vaporisation of the material and $\dot{R} \equiv d R / d t$, where $R(t)$ is the outer radius of the droplet at time $t$.

For mass conservation at the interface, the mass flux out of the liquid phase must equal the mass flux into the gas phase. We represent this algebraically as [2]

$$
C_{v}\left(u_{v}-\dot{R}\right)=-\frac{\rho_{l} \dot{R}}{m_{m}} .
$$

The pressure at the surface of the droplet, due to Assumption 6, is found by generalising the Clapeyron equation to unequal pressures [4]. This gives

$$
\frac{1}{\rho_{l}} \frac{d p_{l}}{d t}-\frac{1}{m_{m} C_{v}} \frac{d p_{v}}{d t}=-\frac{L}{T_{v}} \frac{d T_{v}}{d t}
$$


where the full time derivative is

$$
\frac{d}{d t} \equiv \frac{\partial}{\partial t}+\dot{R} \frac{\partial}{\partial r} .
$$

Considering the balance of forces at the surface leads to an equation for the difference between the gas and liquid pressures at the surface [2]

$$
p_{v}=p_{l}-\frac{\sigma}{R}+\rho_{l} \dot{R} u_{v},
$$

where $\sigma$ is the surface tension, which is taken to be constant. The appearance of $p_{l}$ in this equation is the motivation for using Equation (10).

Initially, the droplet radius is $R_{0}$.

\subsection{Perturbation Analysis}

Nondimensionalisation: We nondimensionalise the system as follows:

$$
\begin{aligned}
t & =t_{0} \tilde{t}, & r & =R_{0} \tilde{r}, & C_{v} & =C_{\infty} \tilde{C}_{v}, \\
u_{v} & =\frac{R_{0}}{t_{0}} \tilde{u}_{v}, & T_{l} & =T_{0} \tilde{T}_{l}, & & T_{v}=T_{\infty} \tilde{T}_{v}, \\
R(t) & =R_{0} \tilde{R}(\tilde{t}), & \dot{R} & =\frac{R_{0}}{t_{0}} \tilde{R}^{\prime}, & & p_{l}=C_{\infty} \bar{R} T_{\infty} \tilde{p}_{l} .
\end{aligned}
$$

All constants in these equations are known except $t_{0}$, which will be chosen in the present work to represent the timescale of evaporation. However, this choice is not unique. Applying these equations leads to the following system (neglecting the tilde notation for simplicity - all variables from here are dimensionless):

At $t=0$,

$$
\begin{aligned}
& T_{l}=1, \\
& T_{v}=1, \\
& C_{v}=1 \text {, } \\
& u_{v}=0 \text {. }
\end{aligned}
$$


At $r=0$,

$$
\frac{\partial T_{l}}{\partial r}=0 .
$$

For $0<r<R(t)$,

$$
\frac{\partial T_{l}}{\partial t}=\nu_{1} \frac{1}{r^{2}} \frac{\partial}{\partial r}\left(r^{2} \frac{\partial T_{l}}{\partial r}\right) .
$$

At $r=R(t)$,

$$
\begin{aligned}
T_{v} & =\nu_{5} T_{l} \\
\frac{\partial T_{v}}{\partial r} & =\nu_{6} \frac{\partial T_{l}}{\partial r}-\nu_{7} \dot{R} \\
C_{v}\left(u_{v}-\dot{R}\right) & =-\nu_{8} \dot{R} \\
\frac{d p_{l}}{d t} & =\nu_{8} \frac{1}{C_{v}} \frac{d\left(C_{v} T_{v}\right)}{d t}-\nu_{9} \frac{1}{T_{v}} \frac{d T_{v}}{d t} \\
C_{v} T_{v} & =p_{l}-\nu_{10} \frac{1}{R}+\nu_{11} \dot{R} u_{v} .
\end{aligned}
$$

For $r>R(t)$,

$$
\begin{aligned}
\frac{D C_{v}}{D t} & =-\frac{C_{v}}{r^{2}} \frac{\partial}{\partial r}\left(r^{2} u_{v}\right) \\
C_{v} \frac{D u_{v}}{D t} & =-\nu_{2} \frac{\partial}{\partial r}\left(C_{v} T_{v}\right) \\
C_{v} \frac{D T_{v}}{D t} & =\nu_{3} \frac{1}{r^{2}} \frac{\partial}{\partial r}\left(r^{2} \frac{\partial T_{v}}{\partial r}\right)-\nu_{4} \frac{C_{v} T_{v}}{r^{2}} \frac{\partial}{\partial r}\left(r^{2} u_{v}\right) .
\end{aligned}
$$

As $r \rightarrow \infty$,

$$
\begin{aligned}
& T_{v} \rightarrow 1 \\
& C_{v} \rightarrow 1
\end{aligned}
$$

Finally, $R(0)=1$.

Oberman [8] gives the exact form of the dimensionless parameters $\nu_{1}$ through $\nu_{11}$ in the above equations. 
Perturbation Analysis: hereafter we consider the fluid to be water. The remaining constants are taken to be within the ranges $273.15 \mathrm{~K} \leq T_{0} \leq$ $423.15 \mathrm{~K}, 323.15 \mathrm{~K} \leq T_{\infty} \leq 423.15 \mathrm{~K}, 1 \mathrm{~mol} \leq C_{\infty} \leq 20 \mathrm{~mol}$, and $1 \mu \mathrm{m} \leq$ $R_{0} \leq 100 \mu \mathrm{m}$. Additionally, $t_{0}$ is taken to be the value that sets $\nu_{7}=1$. As mentioned earlier, this means that $t_{0}$ represents the time scale of evaporation of the droplet.

This then leads to the following ranges for the dimensionless parameters

$$
\begin{aligned}
& 46 \leq \nu_{1} \leq 72 \quad 2.2 \cdot 10^{10} \leq \nu_{2} \leq 2.8 \cdot 10^{14} \quad 7900 \leq \nu_{3} \leq 2.1 \cdot 10^{5} \\
& 0.247 \leq \nu_{4} \leq 0.247 \quad 0.6 \leq \nu_{5} \leq 1.3 \quad 23.4 \leq \nu_{6} \leq 47.5 \\
& 2800 \leq \nu_{8} \leq 5.6 \cdot 10^{4} \quad 3 \cdot 10^{4} \leq \nu_{9} \leq 8 \cdot 10^{5} \quad 0.01 \leq \nu_{10} \leq 31.3 \\
& 9.8 \cdot 10^{-12} \leq \nu_{11} \leq 2.6 \cdot 10^{-6}
\end{aligned}
$$

We conclude that $1 / \nu_{2}$ is a very small parameter, representing the ratio of kinetic energy density to pressure, and can be neglected in a first order perturbation expansion. Neglecting $1 / \nu_{2}$ in Equation (21) and matching with Equations (23) and (24), we find

$$
C_{v} T_{v}=1 .
$$

This result implies that the system is effectively isobaric. This same result has been found previously for other models [5]. Furthermore, we note from Equation (21) that neglecting $1 / \nu_{2}$ elicits a temporal boundary layer in $u_{v}$; however, the analysis of this effect will not be considered here.

Applying Equation (25) to Equations (20) and (22) and equating gives

$$
\frac{\partial T_{v}}{\partial t}+\frac{1}{r^{2}} \frac{\partial}{\partial r}\left(r^{2} u_{v} T_{v}\right)=\frac{2 \nu_{3}}{1+\nu_{4}} \frac{T_{v}}{r^{2}} \frac{\partial}{\partial r}\left(r^{2} \frac{\partial T_{v}}{\partial r}\right) .
$$

Substituting Equation (25) into Equation (18) and integrating in time gives

$$
p_{l}=1-\nu_{9} \log \frac{T_{v}}{T_{k}}
$$


where $T_{k}$ is the value of $T_{v}$ which sets $p_{l}=1$. Substituting Equation (25) into (19) and matching with (27) gives

$$
\log \frac{T_{v}}{T_{k}}=-\frac{\nu_{10}}{\nu_{9}} \frac{1}{R}+\frac{\nu_{11}}{\nu_{9}} \dot{R} u_{v} .
$$

Equations (14), (26) and (28), with associated boundary and initial conditions (12), (13), (15), (16) and (17), constitute our simplified model for drying a liquid droplet in its own vapour.

\section{$3 \quad$ Numerical Techniques}

In solving this system of equations we first transform the equations inside the droplet using $r=x R(t)$, where $0<x<1$, as this fixes the surface of the droplet at $x=1$. Outside the droplet, we apply $r=x+R(t)$, where $0<x<x_{\max }$, as this fixes the surface of the droplet at $x=1$ while ensuring that grid evolution does not cause loss of data at distances away from the droplet at late times. The meshes are then introduced uniformly in $x$.

The resulting transformed equations are discretised using a fully implicit, non-adaptive control volume method. An upwinding scheme determines the vapour temperature at the node points of the control volumes, and the vapour velocity is discretised on a control volume grid that is offset, by half a control volume, from that used to determine the vapour temperature. The nonlinear term in Equation (26) is linearised by supposing that the temperature is approximately uniform across the control volume, and applying a Newton scheme to the system to ensure realistic convergence.

The resulting Algorithm 1 was implemented in Matlab. 


\section{Algorithm 1:}

while $t<t_{\max }$ and $R(t)>0$ do

while Convergence has not occurred do

Determine $T_{l}$ distribution (using discrete form of equation (14)).

Determine $T_{v}$ distribution (using discrete form of equation (26)).

Approximate $\dot{R}$ (using equation (16)).

Determine $u_{v}$ distribution (using discrete form of equation (22) with (25)).

Find error due to the lagging of $u_{v}$ and $\dot{R}$ for convergence criteria.

\section{end while}

Take time step $(t \leftarrow t+\Delta t)$.

\section{end while}

\section{Results and Conclusions}

Figures 1(a) and (b) show the early and long time evolution, respectively, of the size of a water droplet initially at $350 \mathrm{~K}$ as it is dried in an atmosphere of its own vapour initially at a concentration $C_{\infty}=20 \mathrm{~mol} \mathrm{~m}^{-3}$, in which $T_{\infty}=$ $368 \mathrm{~K}$. Initially, the temperature of the liquid at the surface of the droplet, $T_{l}$, is considerably lower than the temperature, $T_{v}$, of the vapour surrounding it and this induces the boundary layer effect observed in Figure 1(a) in which the droplet undergoes condensation at early times before evaporation takes place. This effect, although not overly significant in the current simulation, can become pronounced under more extreme drying conditions and/or for different liquids, and has been observed experimentally [7], and predicted by other models [5, e.g.].

Figures 1(a) and (b) also depict a second set of results in which the surface tension, $\sigma$, of the water droplet has been set to zero. The two simulations $(\sigma \neq 0$ and $\sigma=0)$ are indistinguishable, and we found this to be true for a wide range of initial temperatures, initial vapour concentrations, and initial 

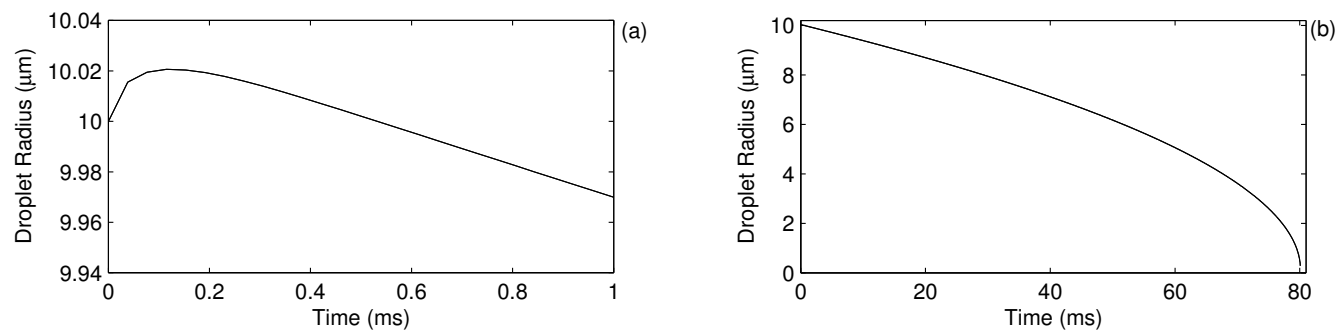

FiguRE 1: Graphs of water droplet radius versus time for (a) early times and (b) long times.

droplet radii. This fact is due to the negligible size of the factor $\nu_{10} / \nu_{9}$. This demonstrates that surface tension plays a negligible role in the evaporation of water droplets in their own vapour.

Godsave [3] observed, empirically, that the radius of a drying liquid droplet is generally of the form $R(t)^{2}=R_{0}^{2}-\beta t$, where $\beta$ is the evaporation constant and $R_{0}$ is the initial droplet radius. Least squares fitting of the data in Figure 1(b) to this function produces a very close match (RMS error of less than $0.2 \%$ ) for a $\beta$ value of $0.0013 \mathrm{~mm}^{2} \mathrm{~s}^{-1}$, thus suggesting that our model may produce results close to those observed experimentally. It is encouraging to see that this model produces such a result, which has been produced many times before by many other models [5, 11, e.g.].

Figures 2(a) and (b) show the evolution of droplet radius and temperature distribution, respectively, at early times, for a water droplet with $T_{0}=T_{\infty}=423 \mathrm{~K}$ and $C_{\infty}=1 \mathrm{~mol} \mathrm{~m}^{-3}$. In contrast with the condensation effect observed in Figure 1(a), we see that in Figure 2(a) the droplet evaporates rapidly. The temperature, $T_{l}$, for the liquid is approximated by the dimensioned form of Equation (28), which after noting that $\nu_{10} / \nu_{9}$ and $\nu_{11}$ are negligible becomes $T_{l} \approx \tilde{T}_{k} T_{\infty}$. In the simulation in Figure 2 , the initial temperature of the droplet is much higher than that predicted by this relation, and as such the liquid temperature drops rapidly during the early stages of drying as shown in Figure 2(b). The heat associated with this loss 

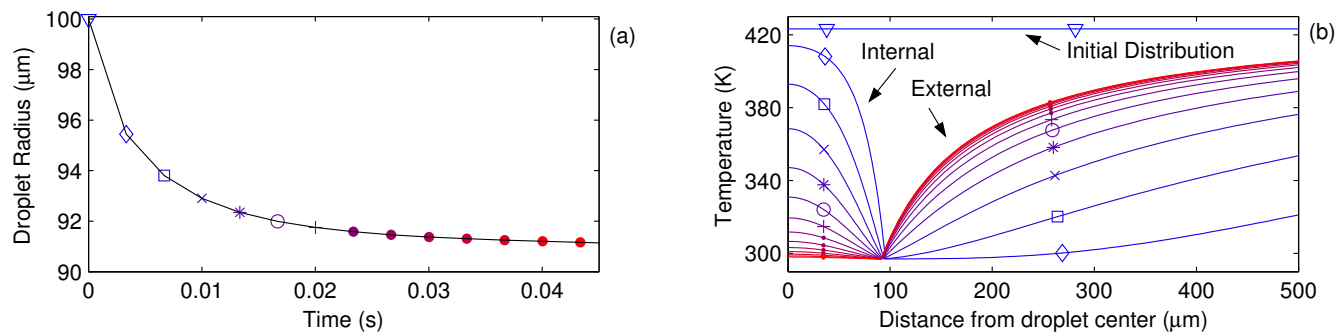

FiguRE 2: Graphs of early time (a) water droplet radius and (b) temperature distributions.

in temperature is used to evaporate the droplet, leading to the profile shown in Figure 2(a).

In conclusion, we note that we have developed a model for the drying of a liquid droplet in its own vapour. The computational algorithm described in Section 3 successfully implements the moving boundary aspect of the problem, and thus the same technique may be applicable to more complex models. We demonstrated via perturbation techniques that the system can be considered to be isobaric, and surface tension was shown to be negligible. The model was found to agree with empirical observations of experimental data.

Acknowledgment: The authors gratefully acknowledge the informative discussions with Prof. C. P. Please of the University of Southampton, Dr I. W. Turner of QUT during the course of this work, and the Australian Institute of Nuclear Sciences and Engineering (AINSE) for financial support.

\section{References}

[1] R. B. Bird, W. E. Stewart, and E. N. Lightfoot. Transport Phenomena. John Wiley and Sons, Inc., Madison, WI, 1960. C1158, C1159 
[2] J. M. Delhaye. Basic Equations for Two-Phase Flow Modeling. In Two-Phase Flow and Heat Transfer in the Power and Process Industries. Hemisphere Publishing Corporation, Washington, DC, 1981. C1160, C1161

[3] G. A. E. Godsave. Studies of the combustion of drops in a fuel spray - the burning of single drops of fuel. Proceedings of the Fourth Symposium (International) of Combustion, pages 818-830, 1953. C1166

[4] G. S. H. Lock. Latent Heat Transfer. Oxford Engineering Science Series. Oxford University Press, New York, NY, 1994. C1160

[5] J. Margerit and O. Sero-Guillaume. Study of the evaporation of a droplet in its stagnant vapor by asymptotic matching. International Journal of Heat and Mass Transfer, 39(18):3887-3898, 1996. http://dx.doi.org/10.1016/0017-9310(96)00051-8 C1158, C1160, C1163, C1165, C1166

[6] K. Masters. Spray drying handbook. Halsted Press, New York, NY, 1979. C1156

[7] H. Nomura, Y. Ujiie, H. J. Rath, J. Sato, and M. Kono. Experimental study on high pressure droplet evaporation using microgravity conditions. Proceedings of the Combustion Institute, 26:1267-1273, 1996. C1165

[8] G. J. Oberman. Unpublished Work, 2004. C1162

[9] A. J. Roberts. A one-dimensional introduction to continuum mechanics. World Scientific, River Edge, NJ, 1994. C1159

[10] Y Sano and R. B. Keey. The drying of a spherical particle containing colloidal material into a hollow sphere. Chemical Engineering Science, 37(6):881-889, 1982.

http://dx.doi.org/10.1016/0009-2509(82)80176-0 C1156 
[11] W. J. Sheu and N. C. Liou. Effect of temporal variation of pressure on vaporization of liquid droplets. International Journal of Heat and Mass Transfer, 42:4043-4054, 1999. http://dx.doi.org/10.1016/S0017-9310(99)00063-0 C1158, C1166

[12] W. A. Sirignano. Fluid Dynamics and Transport of Droplets and Sprays. Cambridge University Press, 1st edition, 1999. C1156

[13] J. van der Lijn. Simulation of heat and mass transfer in spray drying. Centre for Agricultural Publishing and Documentation, Wageningen, 1976. C1156 